Marcin Zatyka

Associação Portuguesa de Ciência Política

\title{
La comunidad ecuménica de Taizé y los cambios políticos en Polonia a fines del siglo XX
}

\section{THE ECUMENICAL COMMUNITY OF TAIZÉ AND POLITICAL CHANGES IN POLAND AT THE END OF THE 20TH CENTURY}

The principal aim of this article is to show the relationship between the Ecumenical Community of Taizé and Poles in the time of political changes in Poland at the end of the 20th century. The Youth Council initiated in the 1970s by the community, which was later transformed into the Young Adults European Meetings, created an opportunity to intensify the ecumenical process, but also to enable meetings of thousands of young Europeans from two antagonistic blocs, eastern and western, separated by the Iron Curtain between 1945 and 1989. Recently published documents of the authorities of the Polish People's Republic (PRL), used by the author in the article, prove hostility of the communist regime towards the Catholic Church in Poland, but also reveal some methods used in this struggle. They also show that shortly before the Youth Council organized in France by the Taizé Community, the communist authorities appointed necessity to conduct actions aimed at weakening the Church in the area of ecumenism and the movements and organizations of lay people, which were slowly developing themselves that time.

The Poles living in the communist state were accompanied by the Taizé brothers. Their dedication have been continued also after the collapse of the regime. The intensity of the events organized by the community has changed, as well as a number of participants of meetings in this French village and the Youth Adults European Meetings organized by Taizé. While during the time of 
communism there were only several dozen or hundred young participants from Poland, after the end of the regime, Poles in the number of tens of thousands dominated these events. During the political transformation Taizé has become one of the main foreign destinations for young Polish Christians, as well as a place of their natural integration with other young adults from the European Union countries. The person who contributed significantly to the inclusion of Poles in the activities of this community, both during the communist period and during the political transformation, was Pope John Paul II form Poland.

Key words: Taizé, ecumenism, Polish People's Republic, communism, European Ecumenism integration, lay people, political transformation in Poland, John Paul II.

La película El Azar de Krzysztof Kieślowski, filmada entre 1980 y 1981, en el corto periodo de apertura en la Polonia comunista, marcado por los huelgas de trabajadores y la fundación del sindicato Solidarnosc, muestra la importancia de relaciones entre los jóvenes cristianos polacos y la comunidad ecuménica de Taizé. ${ }^{1}$ Hasta hoy no hay prácticamente referencias a la utilización por el cineasta polaco del caso de Taizé; por un motivo banal - el actor principal citó erróneamente el nombre del pueblo francés, donde existe la comunidad. Gracias la mala pronunciación del actor, también no fue traducido el nombre de la comunidad ecuménica a los subtítulos, ni a la versión inglesa del película. No obstante $E l$ Azar puede ser reconocido como la primera película que describe la relación entre la comunidad de Taizé y los polacos, en su mayoria jóvenes que viven tras el Telón de Acero, y que participaron en encuentros religiosos en el Europa Occidental. En el guión, escrito por mismo Kieślowski, fue presentada la historia de la vigilancia de los servicios de inteligencia comunista contra un grupo de jóvenes opositores cercanos a los hermanos de la comunidad francesa de Taizé. La escena que presenta el protagonista de la película, Witek Dlugosz, forzado por un funcionario de los servicios secretos comunistas a denunciar extranjeros favorables a la oposición democrática polaca se basó, como demuestran algunos testimonios posteriores y publicaciones, en situaciones reales. ${ }^{2}$

Mediante el chantaje, como la negación de pasaporte, el Ministerio de Interior de la Polonia comunista intentó reclutar no solo a los deportistas o a los artistas que iban a Occidente, sino también a los participantes de eventos religiosos, aparentemente poco significativos. Aunque El Azar fue denunciado por censura de la ley marcial, y su

1 K. Kieślowski, Przypadek (El Azar), 1981.

$2 \quad$ M. Górka, Wywiad z Bratem Markiem z Taizé, en: www.mgorka.prv.pl, 2004. 
presentación tuvo lugar seis años después, todavía durante el periodo comunista, en 1987, el guión de Kieślowski ganó el premio principal del festival del filme polaco de Gdynia. La revelación de las técnicas de los servicios de seguridad comunistas contra los jóvenes asociados a la comunidad de Taizé no alteró prácticamente la estrategia aplicada hasta el final del régimen en emisión de pasaporte. En 2009, durante el juicio de autolustración de Mariusz Handzlik, cuando era subsecretario de Estado en la Cancillería del Presidente de Polonia, fue revelado que intentaron captarlo antes de su viajen a Taizé en 1987. Durante el juicio un ex funcionario del régimen confirmó que los servicios de seguridad comunista chantajearon a Handzlik con el pasaporte. El ex agente explicó que, a pesar de Handzlik negarse a cooperar, fue registrado, de manera falsa o por error, en documentos de inteligencia como colaborador secreto. ${ }^{3}$

Después de junio de 1989, cuando la oposición democrática llegó al poder, Taizé y las reuniones organizadas por esta comunidad se convirtieron en uno de los principales lugares de viaje para miles de jóvenes polacos. Tuvieron la oportunidad de conocer las realidades de los países de Europa Occidental, entre los cuales en aquel tiempo se intensificaron los procesos de integración política, económica y social.

\section{Directrices contra la Iglesia Católica}

La participación de los creyentes polacos en movimientos de la Iglesia, especialmente oriundos del Occidente, fue limitada por las restricciones impuestas por las autoridades de la República Popular de Polonia. Así como en todo el Bloque Soviético. ${ }^{4}$ Además, los obispos polacos no estaban interesados en el desarrollo de grupos religiosos de masa debido al peligro de ser interceptados fácilmente por los servicios de inteligencia comunista. El creciente interés de los creyentes por los movimientos eclesiásticos después del Concilio Vaticano II fue frenado mismo por el primado polaco, el Cardenal Stefan Wyszyński. El Arzobispo de Varsovia, encarcelado durante la época estalinista y bajo vigilancia durante años por los servicios de seguridad, temía sobre la unidad de la Iglesia polaca. ${ }^{5} \mathrm{El}$ temor principal fue una posible infiltración de oficiales y colaboradores de la seguridad comunista a través del laicado a las estructuras de la Iglesia, que en consecuencia

3 Esbek: Mogłemfikcyjniezarejestrować Handzlika, en:www.wprost.pl, 15.07.2009.

M. Zatyka, Un cura que se hizo soviético, "Pregón de La Plata”, Buenos Aires, 01.08.2018, p. 2-3. 
la desestabilizaban. Los documentos publicados recientemente por el Instituto de la Memoria Nacional en Varsovia prueban esas intenciones. El Ministerio del Interior de la República Popular de Polonia (PRL) tuvo planos para debilitar la Iglesia Católica y también otras confesiones religiosas, reconocidas como "antisocialistas debido a sus supuestos doctrinales". 6

En 15 de junio de 1973 fue emitido por el Director del Departamento IV del Ministerio del Interior, una unidad responsable por la lucha contra la Iglesia, un documento llamado "Directrices sobre las formas y métodos de las actividades operacionales del Departamento de IV y sus homólogos en el terreno". En el texto firmado por el subdirector del departamento, Coronel Konrad Straszewski indicó la necesidad de tomar medidas encaminadas a "debilitar la cohesión interna y la eficiencia de las células individuales de la Iglesia". Entre las actividades de desestabilización estaba la propuesta de utilizar las iglesias y las asociaciones religiosas no católicas contra la jerarquía de la Iglesia Católica. Uno de los campos para un posible conflicto entre ellas fue el ecumenismo. El autor de las directrices también señaló la posibilidad de luchar contra la Iglesia a través de los laicos. Él escribió: Desde la posición de las asociaciones de los laicos católicos, hay una gran oportunidad para la deseada influencia sobre el clero y los obispos. ${ }^{7}$

Con la apertura de varios documentos del régimen comunista, se puede suponer que el reconocimiento del ecumenismo y de la actividad de los laicos como áreas que podían debilitar la Iglesia, sirvió al régimen para la justificación de algunas acciones hostiles contra los jóvenes polacos cercanos a Taizé. En junio de 1973, quando fueron publicadas las directrices del Ministerio del Interior polaco, la comunidad de Taizé ya se preparaba intensivamente para el Consejo de la Juventud en Francia. El evento fue inaugurado el 30 de agosto de 1974, reuniendo a más de 40,000 jóvenes, particularmente de los países de la Europa Occidental. ${ }^{8} \mathrm{El}$ encuentro creó una oportunidad para que las autoridades comunistas adquirieran conocimiento de los laicos polacos que estaban en contacto con los cristianos detrás del Telón de Acero y también los nombres de algunos extranjeros simpatizantes de la oposición democrática en Polonia.

$6 \quad$ A.Dziurok, F. Musiał, Instrukcje, wytyczne, pisma Departamentu IV Ministerstwa Spraw Wewnętrznych z lat 1962-1989. Wybór dokumentów, Cracovia - Katowice 2017, p. 33. Ibidem, 561-563. 


\section{El Consejo de Jóvenes y los Encuentros Europeos de Jóvenes}

Polonia, debido al pequeño número de no católicos, no fue un lugar de intenso proceso ecuménico. Pero la creciente popularidad de la comunidad de Taizé creaba una oportunidad para que los jóvenes se fueran al extranjero. Sistemáticamente católicos polacos se unieron al desarrollo de la comunidad fundada en 1940 por el pastor protestante Roger Schutz, llamado hermano Roger. Su objetivo principal fue trabajar con jóvenes cristianos, especialmente de Europa, además de realizar también varias actividades en la área del ecumenismo. Después del Concilio Vaticano II con una autorización de la Santa Sede, los católicos que quisieran podían integrarse a movimientos ecuménicos. En este contexto Taizé comenzó a llamar la atención de muchos jóvenes católicos. Sistemáticamente aumentaba también número de personas que visitaban el pueblo francés. La comunidad mediante pequeños eventos semanales, creó un gran encuentro internacional llamado El Concilio de Jóvenes. La inauguración del evento tuvo lugar en 1974 en Taizé. Más tarde, Roger Schutz realizó varios grandes viajes a distintos países europeos y a otros continentes. Al mismo tiempo se inició la preparación de los documentos del Concilio de Jóvenes. En estos textos, escritos a fines de la década de 1970, fue subrayada la necesidad de renovar las comunidades cristianas a todos los niveles, diocesanos y parroquiales, así como la necesidad de rezar por el Papa. Se colocaron propuestas específicas para la renovación de la liturgia popular y para acercar a los creyentes al misterio de la muerte y la resurrección de Jesucristo a través de la palabra, de la oración y de la Eucaristía. ${ }^{9}$

Con el tiempo, los hermanos de Taizé transformaron el Concilio de Jóvenes en cortas reuniones de pocos días organizadas en varias ciudades de Europa a finales de año. Los eventos llamados "Encuentros Europeos de Jóvenes" inicialmente fueron organizados en países de la Europa Occidental. El primer encuentro tuvo lugar en diciembre de 1978, en París. Otras reuniones de jóvenes animadas por la comunidad de Taizé, en forma de retiros fueron organizadas en los años sucesivos en varias parroquias de Barcelona, Roma y Londres. Los participantes de los encuentros tuvieron la posibilidad de compartir con otros jóvenes su fe y la experiencia de la vida cristiana, así como participar en oraciones. Un elemento importante de los encuentros fueron, desde inicio, pequeños debates sobre la situación de la Iglesia,

E. Weron, Ruchy odnowy we wspótczesnym Kościele, Poznań, 1993, p. 105. 
especialmente en lugares de sufrimiento de los cristianos. No faltaban allí testimonios de personas perseguidas por su fe en países comunistas de la Europa Central y del Este.

El primer gran encuentro entre jóvenes de Polonia y jóvenes del Europa Occidental surgió en 1974 en Taizé durante el Concilio de Jóvenes. A pesar del reducido número de visitantes polacos en los encuentros organizados por la comunidad ecuménica, no faltaban los testimonios sobre la dureza de la vida bajo el régimen. Los hermanos de Taizé también enviaban a Polonia pequeños grupos de jóvenes para reunirse con cristianos polacos, así como con los obispos. Uno de ellos fue el primado de Polonia el Cardenal Stefan Wyszyński. Normalmente los jóvenes de Taizé se juntaron a los peregrinaciones a Czestochowa. ${ }^{10}$

Los primeros encuentros de Taizé organizados en ciudades de Europa no fueron muy importantes para los católicos polacos. Entre 1978 y 1988, en estos eventos participaron sólo pequeños grupos de jóvenes de Polonia, pero ya se creó la oportunidad de reunirse con otras personas de la misma edad y conocer mejor la realidad del cristianismo en Europa Occidental. Para algunas personas de la Polonia comunista los encuentros crearon lazos naturales con otros jóvenes y las comunidades parroquiales detrás del Telón de Acero. ${ }^{11}$

\section{El tiempo del Solidarność}

El periodo de mayor libertad en la Polonia comunista, entre 1980 y 1981, coincidió con la formación del primer sindicato independiente en el Bloque Oriental, llamado Solidarność. En la organización participaron muchos jóvenes, incluido activistas católicos asociados con la Comunidad de Taizé. Algunos de ellos, junto con la imposición de la ley marcial en Polonia el 13 de diciembre de 1981, sufrieron diversas formas de represión. ${ }^{12}$

La ley marcial declarada por el general Wojciech Jaruzelski paró el proceso de democratización de Polonia y aisló al país durante meses del resto del mundo. El cierre de fronteras provocó una dificultad aún mayor para los viajes de los polacos al extranjero. La participación de jóvenes polacos en los encuentros europeos animados por hermanos de la Comunidad de Taizé también se vio perjudicada. Un año antes, en diciembre de 1980, alrededor de 600 jóvenes polacos lograron llegar a la reunión en Roma. Al mismo tiempo tuvieron la oportunidad de

\footnotetext{
$10 \quad$ Brat Roger z Taizé, Jego miłość jest ogniem, Katowice, 1992, p. 98-99.

11 M. Zatyka, M. Zatyka, Ekumeniczna Wspólnota z Taizé, Ząbki 1999, p.72-75.

$12 \quad$ Ibidem, p. 72.
} 
conocer a Juan Pablo II. Según algunas fuentes fue el mismo Papa quien ayudó a negociar con el estado comunista para facilitar el viaje de los jóvenes polacos..$^{13}$ El Presidente del Episcopado Polaco para el Ecumenismo, Obispo Wladyslaw Miziolek ayudó a organizar los primeros viajes. Mencionó que las autoridades de la República Popular de Polonia no eran muy receptivas a estas peregrinaciónes y causaban dificultades. El Ministerio del Interior estaba particularmente perjudicando a los jóvenes al no concederles el pasaporte. ${ }^{14}$

Desde el principio de los Encuentros Europeos de Jóvenes en 1978, el hermano Marek fue el único polaco en la Comunidad de Taizé durante el periodo comunista, ayudando a mantener el contacto con los jóvenes polacos. Él mismo sufrió las dificuldades provocadas por las autoridades comunistas. Antes de ser admitido en la comunidad en 1977, visitó el pueblo francés dos veces, participando, entre otros, en el Concilio de la Juventud. Cuando decidió unirse a la comunidad, el Ministerio Interior polaco rechazó su pasaporte durante dos años. ${ }^{15}$

Los mayores problemas para abandonar el país, los tuvieron los católicos polacos que se inscribieron en el Encuentro Europeo de Jóvenes de Londres en diciembre de 1981. La ley marcial anunciada el 13 de diciembre evitó que los autocaros cruzaran la frontera polaca. En señal de solidaridad con los jóvenes polacos, durante la reunión en Londres, los jóvenes europeos cantaron en polaco la canción "Canta al Señor, toda la tierra". ${ }^{16}$

Debido a los problemas que tenían jóvenes polacos para viajar al oeste europeo, los hermanos de Taizé decidieron tomar la iniciativa y de vez en cuando venían discretamente a Polonia. El hermano Roger también vino varias veces. Llegó en Mayo de 1981 para participar en el funeral del cardenal Stefan Wyszyński en Varsovia. Al mismo tiempo se reunió con jóvenes polacos, de quienes recibió un ramo de flores para el Papa Juan Pablo II hospitalizado en la clínica romana de Gemelli, después del atentado del 13 de Mayo de 1981. El fundador de Taizé fue recibido por el Papa polaco a pesar de riguroso régimen de visitas del herido Papa.

13 J. Bątkiewicz-Brożek, Do Taizé coraz bliżej, “Tygodnik Powszechny”, Cracovia 51-52/2009.

$14 \quad$ M. Zatyka, M. Zatyka, Ekumeniczna Wspólnota..., p. 71-72.

$15 \quad$ M. Górka, Wywiad...

$16 \quad$ M. Zatyka, M. Zatyka, Ekumeniczna Wspólnota..., p. 72. 


\section{Los líderes de la Iglesia polaca y Taizé}

La jerarquía de la Iglesia Católica de Polonia tenía relaciones cercanas con Taizé. El Papa polaco Juan Pablo II tuvo un papel importante en el acercamiento de ambos lados. Hasta principios de los años 80, una parte del clero y del episcopado polaco mantuvieron la distancia con esta comunidad ecuménica. La desconfianza acabó con la decisión de Juan Pablo II de invitar a los hermanos de Taizé a Roma. El propio Papa participó en las oraciones de hasta tres encuentros europeos organizados en la capital italiana en la década de los 80. Hasta el final Ecumenism de su vida Juan Pablo II enviaba todos los años una carta con mensaje dirigido a los participantes de estos encuentros, a los organizadores y a las familias de acogida. ${ }^{17}$

En realidad Karol Wojtyła fue el primer padre polaco que promovió la comunidad de Taizé. Su encuentro con Roger Schutz y otros hermanos tuvo lugar en 1962 durante las sesiones del Concilio Vaticano II, en Roma. En 1964 el Arzobispo de Cracovia visitó Taizé y posteriormente regresó cuatro años después. También recibía al hermano Roger cuando éste visitaba Polonia. El más importante gesto de Juan Pablo II con la comunidad ecuménica, fue su peregrinación a Taizé en 1986, durante una visita oficial a Francia.

La simpatía de los hermanos de Taizé fue correspondida por parte de Karol Wojtyła y sus compatriotas. El hermano Roger durante el régimen comunista llegó a visitar a Polonia muchas veces. A menudo paraba en casas de miembros del Club de la Inteligencia Católica (KIK), como por ejemplo el periodista del semanario "Tygodnik Powszechny", Jerzy Turowicz. El prior de la comunidad mantuvo buenas relaciones con los respectivos clubes: de Varsovia y Cracovia, apoyando las iniciativas de estas organizaciones. ${ }^{18}$ De KIK salieron varios políticos de la oposición, que a comienzos de la década de los 90 iniciaron a construir las bases de la Tercera República de Polonia.

Las relaciones de la jerarquía de la Iglesia Católica de Polonia con la Comunidad Ecuménica de Taizé con el tiempo se fortalecieron. Un gran acercamiento tuvo lugar en los últimos años del comunismo. En 1986 el Senado de la Academia de Teología Católica en Varsovia, llamado desde 1999 el Universidad de Cardenal Stefan Wyszyński de Varsovia (UKSW), llegó a entregar al hermano Roger, el fundador de la Comunidad de Taizé, el doctorado honoris causa. En ese mismo año, a finales de octubre, poco después de la visita del Papa Juan Pablo II

17 M. Zatyka, La entrevista con el hermano José Maria Ribeiro OP. En posesión del autor.

18 S. Oder, S. Gaeta, Joao Paulo II Santo, Lisboa 2010, p. 73. 
a Taizé, la villa francesa fue visitada por más de una docena de obispos polacos, que incluyó también al cardenal de Wrocław, Henryk Gulbinowicz. Los obispos polacos pararon en Taizé dos días y dos noches en el camino de regreso del Santuario de Lourdes. Aún seis meses antes, en abril de 1986, llegó a la Comunidad Ecuménica de Taizé el Primado de Polonia, Cardenal Jozef Glemp, con el Obispo Stefan Wesoly, que en aquel momento era responsable del servicio pastoral a los polacos que vivían en el extranjero. ${ }^{19}$

Los miembros de la Conferencia Episcopal de Polonia desempeñaron un papel importante en la organización de viajes a Taizé y a los Encuentros Europeos de Jóvenes celebrados en los países de Europa occidental en los últimos años del comunismo. No sólo facilitaron el proceso de alquilar autobuses estatales para las peregrinaciones a Taizé, sino que también rellenaron y firmaron documentos necesarios en los procedimientos de solicitud de pasaportes expedidos por las autoridades comunistas de la República Popular de Polonia. ${ }^{20}$

\section{El encuentro con el Occidente}

El apoyo de los obispos polacos para promover las ideas de reconciliación y unidad entre los cristianos, promovidas por los hermanos de Taizé también se manifestaba en acciones organizadas en el territorio polaco, por ejemplo en el apoyo recibido durante los encuentros europeos en Wrocław y Varsovia. La elección de Wrocław, como ciudad anfitriona de Encuentro Europeo de Jóvenes en 1989, fue uno de los momentos decisivos en la relación con los católicos polacos. La pequeña comunidad francesa gracias al encuentro se tornó famosa para millares de ciudadanos de Polonia, tanto cristianos como no creyentes. Entre las multitudes que participaron en el evento con los hermanos de Taizé, transmitido por los medios de comunicación social, se encontraban importantes personajes de la Iglesia polaca y del Estado, entre ellos el presidente de gobierno Tadeusz Mazowiecki.

En el siglo XX la comunidad ecuménica de Taizé organizó en Polonia tres encuentros europeos para jóvenes. En Wrocław la iniciativa se desarrolló dos veces: en 1989 y 1995, y en Varsovia una vez, en 1999. Durante los encuentros todos los participantes pasaron el evento hospedados en casas de familias. Anteriormente, en otras ciudades europeas, la comunidad nunca consiguió encontrar sitios suficientes, usando algunas escuelas y centros parroquiales. De media, en cada

$19 \quad$ M. Zatyka, M. Zatyka, Ekumeniczna Wspólnota..., p. 73-75.

20 G. Koneczna, Taizé w Polsce, en: https://www.taize.fr/pl_article452.html 
uno de estos eventos participaron más de 50.000 de personas. Del mismo modo, fue muy fuerte la presencia de los jóvenes polacos en los encuentros europeos organizados por la comunidad de Taizé a partir de 1990, es decir después del comunismo. Estos jóvenes, venidos de todas las regiones del país, representaban el grupo más numeroso de los que participaba en los Encuentros Europeos de Jóvenes. Muchos de ellos no siempre tenían vínculos a sus comunidades parroquiales ni antes habían realizado tareas para la Iglesia. ${ }^{21} \mathrm{El}$ año récord fue 1992, cuando para el encuentro en Viena se desplazaron más de 60 mil polacos, dominando las estadísticas de los participantes de este evento. En los años siguientes el número de participantes oriundos de Polonia bajó, pero hasta el final del siglo XX nunca vinieron menos de 30 mil polacos. Para todos ellos, la peregrinación al Encuentro Europeo de Jóvenes comenzaba en los puntos de preparación, especialmente en las parroquias. Desde el inicio de la Tercera República de Polonia en todo el país funcionaban más de 200 puntos de acogida, formados por grupos a menudo vinculados a la Iglesia Católica, así como a las varias Iglesias Protestantes. ${ }^{22}$

Durante los encuentros europeos, todos los jóvenes fueron invitados a participar en actividades sociales y de caridad, así como charlas sobre la unidad y la reconciliación entre las naciones. El propio fundador de la Comunidad de Taizé, el hermano Roger, en uno de sus discursos durante el Encuentro Europeo de Jóvenes en París, en el que participaron 50.000 jóvenes de Polonia, los alentó a realizar tareas en la sociedad europea. - Así, muchos jóvenes polacos que conocemos, viven la primera bendición: felices son los pobres de corazón, porque ellos ver a Dios, y ellos ya ven algo del misterio de Dios. Tengo la impresión de que en el alma polaca hay sólo la simplicidad, no hay ninguna pretensión espiritual. Vosotros sois entonces aquellos felices que -como muchos antes de vosotros - quedarán en el corazón de Europa como una levadura de confianza, de paz y de reconciliación. De esta forma, pueden construir Europa - dijo a los jóvenes polacos hermano Roger de Taizé. ${ }^{23}$

A partir de 1990 los jóvenes polacos participaron cada vez más en los encuentros organizados cada semana en el pueblo de Taizé, en Borgoña. En la década de los 90 llegaron allí miles de ciudadanos de un país en fase de transición. Para muchos de ellos el viaje a Taizé fue como una primera oportunidad de salir del país, aislado durante 50

\footnotetext{
21 E. Weron, Ruchy odnowy..., p. 104-106.

22 G. Koneczna, Taizé $w .$.

23 Ibidem.
} 
años de guerra y comunismo. Las visitas a la comunidad que promovía la reconciliación entre los pueblos de Europa y la colaboración mutua entre las naciones fueron, de hecho, el primer paso de la apertura a la idea de integración europea. ${ }^{24}$ En los encuentros semanales en Taizé participaron no sólo cristianos adultos de varios países de Europa, sino también políticos famosos y altos funcionarios estatales. En el marco de una visita privada viajaron a Taizé los ex diputados Andrzej Wielowieyski y Gabriel Janowski, así como el ex presidente Bronislaw Komorowski.

Las actividades de la Comunidad Ecuménica de Taizé, en el marco de la animación de encuentros internacionales, se profundizaron con el impulso dado por Juan Pablo II en Polonia a la idea de las Jornadas Mundiales de la Juventud. El proyecto fue creado en base a la experiencia de los Encuentros Europeos de Jóvenes organizados desde 1978. Los encuentros con el Papa comenzaron en 1986 en Roma y se extendieron a otros continentes, limitándose a las personas de ambientes católicos. En ambos casos, los eventos sirvieron para movilizar a los jóvenes polacos a viajar y participar en las reuniones internacionales y para lograr una mayor apertura al mundo. Desde el inicio de Jornadas Mundiales de Jóvenes, los hermanos de Taizé se comprometieron a ayudar en la organización de estos eventos. Uno de ellos tuvo lugar en 1991 en Polonia, en Czestochowa, donde vinieron más de 1,6 millones de personas. ${ }^{25}$

La comunidad de Taizé es una de las pocas organizaciones occidentales que al inicio del período de transición democrática en Polonia invitaba a los católicos de este país a tener una actitud más abierta hacia los extranjeros y a fortalecer los vínculos con la Iglesia universal. Por otra parte, Taizé propuso una forma menos exigente y menos implicada en la vida de la Iglesia estructural, que la Acción Católica restaurada en Polonia en 1996. A diferencia de otros grupos activos en la Iglesia Católica, las iniciativas tomadas por los hermanos de la comunidad ecuménica en las parroquias y capellanías universitarias polacas no tenían fuertes lazos a la estructura eclesiástica. Esta relativamente independiente forma de evangelización de jóvenes contribuyó a la atracción de varios adolescentes alejados de la Iglesia Católica. Muchos de ellos antes de conocer a Taizé nunca habían tenido una cercanía con la vida espiritual, siendo selectivos en sus convicciones religiosas o simplemente no estando vinculados a su parroquia. Gracias

24 M. Zatyka, João Paulo II e a integração polaca na Europa unida, Lisboa 2015, p. 236-238.

25 Centrum Myśli Jana Pawła II, nauczanie jp2.pl 
a Taizé muchos polacos tuvieron oportunidad de no sólo participar en la preparación de encuentros ecuménicos, sino también realizar tareas evangelizadoras y sociales. Los participantes reunidos en los Encuentros Europeos de Jóvenes antes y durante el evento se quedaron en la vida de la parroquia, asumiendo varias responsabilidades sociales. ${ }^{26}$ A través de esta experiencia podían mutuamente descubrir la riqueza de sus iglesias locales y también derribar estereotipos y desconfianza hacia los ciudadanos de los países de la antigua Comunidad Económica Europea. La experiencia de Taizé no dejó a los jóvenes polacos indiferentes. Volvían a Polonia con la experiencia de la universalidad de la Iglesia y la apertura a los países de Europa Occidental, que durante muchos años quedaron al otro lado del Telón de Acero. ${ }^{27}$ Hasta algunos críticos del papel de la comunidad ecuménica el Igreja apuntan a su popularidad y universalidad en el espacio eclesiástico y público polaco. ${ }^{28}$ En varios casos la comunidad de Taizé creó el primer contacto con la Iglesia y envolvió en la actividad a otros movimientos religiosos. En Polonia no faltaban entretanto intentos de otros grupos, tanto religiosos, como no religiosos, de copiar experiencias de la comunidad fundada por el hermano Roger. El fundador de encuentros de jóvenes en Lednica, el padre Jan Góra confirmaba las raíces de su proyecto en la comunidad de Taizé, ${ }^{29}$ en el cual hoy participan anualmente más de 100.000 jóvenes. Taizé inspiró también al activista social Marek Kotański, cuando fundó los primeros núcleos del Movimiento de Corazones Puros ${ }^{30}$, para lucha contra las adicciones.

\section{Conclusiones}

Polonia, a fines del siglo XX, fue sujeto a un proceso de cambio político basado en la democratización del Estado comunista tras el Telón de Acero. Desde la década de 1970 los hermanos de la comunidad ecuménica de Taizé intentaron "abrir" este país cerrado a través del Consejo de la Juventud y los Encuentros Europeos de Jóvenes. Estos eventos propiciaron una oportunidad para que los jóvenes polacos viajaron y conocieran los países del Bloque Occidental y a los cristianos que vivían allí. A la luz de los documentos del régimen descubiertos

L. M. Jakoniuk, Taizé - miejscem krzyżowania się ludzkich dróg. Fenomen spotkan, "Katecheta", 12/2009, p. 32-39.

27 G. Koneczna, Taizé $w$...

J. Jenkins, Cała prawda o wspólnocie z Taizé, "Zawsze wierni”, 1-2014 (170).

La apresentación Lednica 2000. Poznań, 2015. 
últimamente, las autoridades comunistas de Polonia trataron de reclutar colaboradores secretos entre los jóvenes que asistían a los encuentros organizados por la comunidad ecuménica de Taizé. Durante muchos años, una de las pocas referencias a esta práctica fue la película "El Azar" de Krzysztof Kieślowski. La comunidad de Taizé, como muestra el filme, no solo creó las condiciones para unos encuentros de jóvenes de dos bloques antagónicos, sino también formaba y acompañaba a régimen, algunos de ellos, incluidos miembros de los Clubes Intelectuales Católicos, cercanos a Taizé, se convirtieron en parte de la nueva elite política. Finalmente, Taizé, que en sus inicios fue una comunidad poco conocida, visitada por pequeños grupos de Polonia, se convirtió en el animador de grandes eventos en los que participaron más de 50,000 polacos. Los encuentros integraron jóvenes de ambos lados de Europa. Los participantes polacos de estas reuniones también tuvieron una oportunidad de abrirse a la idea del ecumenismo, así como al proceso de integración de Polonia en la Unión Europea, processo que culminó en 2004.

\section{EKUMENICZNA WSPÓLNOTA Z TAIZÉ A ZMIANY POLITYCZNE W POLSCE KOŃCA XX WIEKU}

Celem niniejszego artykułu jest ukazanie relacji między ekumeniczną Wspólnotą z Taizé a Polakami na tle zmieniającej się sytuacji politycznej w Polsce końca XX w. Zainicjowany w latach 70. przez tę wspólnotę Sobór Młodych, który następnie przekształcił się w Europejskie Spotkania Młodych, stworzył okazję nie tylko do intensyfikacji procesu ekumenicznego, ale też do regularnych spotkań tysięcy młodych Europejczyków z antagonistycznych bloków, wschodniego i zachodniego, rozdzielonych Żelazną Kurtyną między 1945 a 1989 r. Upublicznione w ostatnim czasie dokumenty władz Polskiej Rzeczpospolitej Ludowej (PRL), z których korzystał autor, dowodzą nie tylko wrogości komunistycznego reżimu wobec struktur Kościoła katolickiego w Polsce, ale też ujawniają stosowane w tej walce metody. Ukazują również, iż na krótko przed organizowanym we Francji przez Wspólnotę z Taizé Soborem Młodych władze komunistyczne uznały za niezbędne akcje w obszarze ekumenizmu oraz rozwijających się dopiero w PRL ruchów i organizacji katolików świeckich, służące osłabieniu Kościoła. Solidarni z żyjącymi w komunistycznym państwie Polakami bracia z Taizé nie ustali w animowaniu młodzieżowych spotkań także po upadku reżimu. Zmieniła się intensywność tych wydarzeń, a także liczba osób wyjeżdżających do tej francuskiej miejscowości i na organizowane przez Taizé Europejskie Spotkania Młodych. O ile w okresie PRL udawało się 
tam od kilkudziesięciu do kilkuset młodych katolików, o tyle po zakończeniu komunistycznego reżimu Polacy w liczbie kilkudziesięciu tysięcy osób rocznie zdominowali te wydarzenia. W okresie transformacji ustrojowej Taizé stało się jednym z głównych miejsc zagranicznych wyjazdów grup młodych chrześcijan z Polski, a także ich naturalnego integrowania się z rówieśnikami z państw Unii Europejskiej. Osobą, która w znaczący sposób przyczyniła się do włączenia Polaków w działalność tej wspólnoty, zarówno w okresie komunizmu, jak i podczas transformacji ustrojowej, był pochodzący z Polski papież Jan Paweł II.

Słowa kluczowe:Taizé, ekumenizm, Polska Rzeczpospolita Ludowa, komunizm, Ecumenism integracja europejska, laikat, transformacja ustrojowa w Polsce, Jan Paweł II.

\section{Bibliografía:}

1. Brat Roger z Taizé, Jego miłość jest ogniem, Katowice, 1992.

2. Brat Roger z Taizé, Niech twoje święto trwa bez końca, Warszawa 1982.

3. Bątkiewicz-Brożek J., Do Taizé coraz bliżej, "Tygodnik Powszechny", Cracovia 51-52/2009.

4. Dziurok A., Musiał F., Instrukcje, wytyczne, pisma Departamentu IV Ministerstwa Spraw Wewnętrznych z lat 1962-1989. Wybór dokumentów, Cracovia - Katowice 2017.

5. Esbek: Mogtem fikcyjnie zarejestrować Handzlika, en: www.wprost.pl, 15.07.2009.

6. Górka M., Wywiad z Bratem Markiem z Taizé, en: www.mgorka.prv.pl, 2004.

7. Jakoniuk L. M., Taizé - miejscem krzyżowania się ludzkich dróg. Fenomen spotkan, "Katecheta", 12/2009, p. 32-39.

8. Jenkins J., Cała prawda o wspólnocie z Taizé, "Zawsze wierni”, 1-2014 (170).

9. Kieślowski K., Przypadek (El Azar), 1981.

10. Koneczna G., Taizé w Polsce, en: https://www.taize.fr/pl_article452.html

11. Lasota M., Donos na Wojtyte, Cracovia, 2006.

12. Oder S., Gaeta S., Joao Paulo II Santo, Lisboa 2010.

13. Weron E., Ruchy odnowy we wspótczesnym Kościele, Poznań, 1993.

14. Zatyka M., João Paulo II e a integração polaca na Europa unida, Lisboa 2015.

15. Zatyka M., La entrevista con el hermano José Maria Ribeiro OP. En posesión del autor.

16. Zatyka M., Un cura que se hizo soviético, "Pregón de La Plata", Buenos Aires, 01.08.2018, p. 2-3.

17. Zatyka M., Zatyka M., Ekumeniczna Wspólnota z Taizé, Ząbki 1999.

18. Zatyka M., Zatyka M., O tym, co najważniejsze, Varsovia 2004. 